\title{
A PROJECTION-BASED SEMI-BLIND CHANNEL ESTIMATION FOR LONG-CODE WCDMA
}

\author{
Youngchul Sung and Lang Tong \\ School of Electrical and Computer Engineering \\ Cornell University \\ Email: \{ys87,ltong $@$ ee.cornell.edu
}

\begin{abstract}
The third generation $(3 \mathrm{G})$ wireless systems such as Wideband CDMA(WCDMA) employ coherent detection where pilot symbols for channel estimation are transmitted simultaneously with data using orthogonal channelization codes. The orthogonality of codes, unfortunately, is lost easily due to multipath delays. Furthermore, the use of long scrambling code introduces time variation in channel model. In this paper, based on the projection of time-varying subspaces, we present a semi-blind channel estimation technique for long code CDMA systems that use code division multiplexed pilot symbols.
\end{abstract}

\section{INTRODUCTION}

Code division multiple access(CDMA) systems such as Wideband CDMA have been adopted as the standard of the third generation mobile communication systems that are designed to support multimedia communication with high data rate and variable quality of service. One of the new features is coherent detection in the reverse link [4] where pilot symbols are code division multiplexed in the quadrature phase and transmitted with the traffic data simultaneously [3]. In such systems, channel estimation plays a crucial role to improve the overall performance.

In the absence of multipath delays, the orthogonality of the channelization code makes it possible to separate training from data symbols, and conventional training-based channel estimators can be used directly. The presence of multipath delays, unfortunately, destroys the assumed orthogonality. As a result, the accuracy of training-based estimators is severely limited by the cross interference between data and pilot symbols. The performance degradation is especially pronounced for high rate systems that have low spreading gain and when unbalanced power allocations that favor data are used. For such systems, semi-blind channel estimators which utilize both training and data symbols are appropriate. Mestre and Fonollosa analyzed the asymptotic performance of maximum likelihood estimation for WCDMA systems with several assumptions on data using a randomized code approach, which shows that semiblind methods outperform the training-only estimation [5].

While the gain of semi-blind channel estimator is justified theoretically [2], finding effective estimation algorithms remains a major challenge. The maximum likelihood (ML) based approaches, despite their asymptotic optimality, suffer from the presence of local optima and prohibitive complexity. For long code WCDMA, closed-form semi-blind channel estimators are missing currently.

In this paper, we propose a new semi-blind channel estimation method using orthogonal projections by exploiting a special structure of long code WCDMA. As a closed-form batch algorithm, the proposed algorithm effectively eliminates cross interference between data and pilot symbols, resulting in a considerable gain over training based techniques at medium to high signal-to-noise ratios.

The paper is organized as follows. The data model of a CDMA system is described in section 2. A semi-blind channel estimation method based on projection is proposed with reasonable assumption in section 3 . In section 4 , the performance of the proposed method is assessed by Cramér-Rao bound and Monte Carlo simulations in additive Gaussian and frequency selective fading channel. Possible extensions to the multiuser case are discussed in section 5 .

\section{DATA MODEL}

We assume different long scrambling codes are assigned to separate users, and short orthogonal codes are used for channelization of pilot and data symbols. As illustrated in Fig. 1, the data sequence $s_{d}[m]$ is assigned to the in-phase component and the control sequence $s_{p}[\mathrm{~m}]$ to the quadrature phase component. Data and control channels are spreaded by orthogonal channelization code $c_{d}$ and $c_{p}$. A userspecific long complex scrambling code $c_{s, 1}$ is applied next.

We assume that the channel is constant over one slot period and has an $L$-tap finite impulse response (FIR). The multipath delays $\tau_{k}$, much less than the symbol interval, are multiples of chip duration $T_{c}$. We further assume that the 
base station has acquired timing, and the signal is sampled at the chip-rate followed by a descrambler.

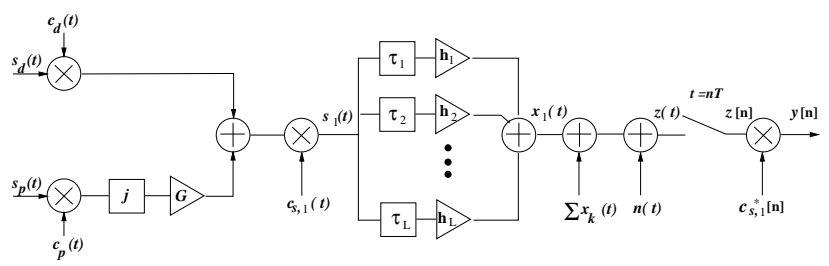

Fig. 1. System model

Channel estimation is performed based upon $M$ pilot symbols within one slot. Stacking the chip-rate descrambled samples and removing those corrupted by the data from the previous slot, we obtain

$$
\mathbf{y}=\mathcal{T}\left(\mathbf{s}_{p}\right) \mathbf{h}+\mathcal{H}(\mathbf{h}) \mathbf{s}_{d}+\mathbf{n}
$$

where $\mathbf{y}$ is the descrambled observation vector, $\mathbf{h}=\left[h_{1}, \ldots, h_{L}\right]^{T}$ the $L \times 1$ complex channel vector, $\mathbf{s}_{p}=\left[s_{p}[1], \ldots, s_{p}[M]\right]^{T}$ the $M \times 1$ pilot symbol vector, $\mathbf{s}_{d}=\left[s_{d}[1], \ldots, s_{d}[M]^{T}\right.$ the $M \times 1$ unknown data symbol vector, $\mathcal{T}\left(\mathbf{s}_{p}\right)$ the known data matrix generated from pilot symbols, $\mathcal{H}(\mathbf{h})$ the unknown filtering matrix, and $\mathbf{n}$ the proper complex Gaussian noise vector with zero mean and variance $\sigma_{n}^{2} I$. It is also assumed that the pilot, data, and noise signals are uncorrelated to each other. Due to the code division multiplexing, the received signal has components from training and data simultaneously. The first term in eq.(1) represents the training part of the received signal. It is necessary to exploit the structure of the model given in eq.( 1). Let $T_{c}$ be the chip interval, $N_{s}$ the spreading gain, $\Delta_{k} \triangleq \tau_{k} / T_{c}$ the delay of the $k$-th path, and $G$ the pilot gain. The $\left((M-1) N_{s}+N_{s}-\Delta_{L}\right) \times L$ data matrix $\mathcal{T}\left(\mathbf{s}_{p}\right)$ can then be expressed as

$$
\mathcal{T}\left(\mathbf{s}_{p}\right)=\left(\begin{array}{c}
\tilde{\mathcal{T}}_{1}\left(\mathbf{s}_{p}\right) \\
\mathcal{T}_{2}\left(\mathbf{s}_{p}\right) \\
\vdots \\
\mathcal{T}_{M}\left(\mathbf{s}_{p}\right)
\end{array}\right)
$$

where $\tilde{\mathcal{T}}_{1}\left(\mathbf{s}_{p}\right)$ is a $\left(N_{s}-\Delta_{L}\right) \times L$ submatrix expressed as

$\tilde{\mathcal{T}}_{1}\left(\mathbf{s}_{p}\right)=j G\left(\begin{array}{lll}c_{p}\left[\Delta_{L}+1\right] s_{p}[1] & \ldots & \gamma_{1, L}\left[\Delta_{L}+1\right] c_{p}[1] s_{p}[1] \\ c_{p}\left[\Delta_{L}+2\right] s_{p}[1] & \ldots & \gamma_{1, L}\left[\Delta_{L}+2\right] c_{p}[2] s_{p}[1] \\ \vdots & \vdots & \vdots \\ c_{p}\left[N_{s}\right] s_{p}[1] & \ldots & \gamma_{1, L}\left[N_{s}\right] c_{p}\left[N_{s}-\Delta_{L}\right] s_{p}[1]\end{array}\right)$

and $\gamma_{1, k}[n] \triangleq c_{s, 1}^{*}[n] c_{s, 1}\left[n-\Delta_{k}\right]$ which results from descrambling the long code. For $2 \leq m \leq M, \mathcal{T}_{m}\left(\mathbf{s}_{p}\right) /(j G)$ is a $N_{s} \times L$ submatrix as

$$
\left(\begin{array}{lll}
c_{p}[1] s_{p}[m] & \ldots & \gamma_{1, L}\left[n_{m}+1\right] c_{p}\left[N_{s}-\Delta_{L}+1\right] s_{p}[m-1] \\
\vdots & \vdots & \vdots \\
c_{p}\left[\Delta_{L}\right] s_{p}[m] & \ldots & \gamma_{1, L}\left[n_{m}+\Delta_{L}\right] c_{p}\left[N_{s}\right] s_{p}[m-1] \\
c_{p}\left[\Delta_{L}+1\right] s_{p}[m] & \cdots & \gamma_{1, L}\left[n_{m}+\Delta_{L}+1\right] c_{p}[1] s_{p}[m] \\
\vdots & \ldots & \vdots \\
c_{p}\left[N_{s}\right] s_{p}[m] & \ldots & \gamma_{1, L}\left[m N_{s}\right] c_{p}\left[N_{s}-\Delta_{L}\right] s_{p}[m]
\end{array}\right)
$$

where $n_{m}=(m-1) N_{s}$. The $\left((M-1) N_{s}+N_{s}-\Delta_{L}\right) \times M$ filtering matrix $\mathcal{H}(\mathbf{h})$ is block bidiagonal and expressed as

$$
\mathcal{H}(\mathbf{h})=\left(\begin{array}{cccc}
\tilde{\mathbf{h}}_{2,1} & & & \\
\mathbf{h}_{1,2} & \mathbf{h}_{2,2} & & \\
& \ddots & \ddots & \\
& & \mathbf{h}_{1, M} & \mathbf{h}_{2, M}
\end{array}\right)
$$

where $\tilde{\mathbf{h}}_{2,1}$ is an $\left(N_{s}-\Delta_{L}\right)$-column vector

$$
\tilde{\mathbf{h}}_{2,1}=\left(\begin{array}{c}
c_{d}\left[\Delta_{L}+1\right] h_{1}+\ldots+\gamma_{1, L}\left[\Delta_{L}+1\right] c_{d}[1] h_{L} \\
c_{d}\left[\Delta_{L}+2\right] h_{1}+\ldots+\gamma_{1, L}\left[\Delta_{L}+2\right] c_{d}[2] h_{L} \\
\vdots \\
c_{d}\left[N_{s}\right] h_{1}+\ldots+\gamma_{1, L}\left[N_{s}\right] c_{d}\left[N_{s}-\Delta_{L}\right] h_{L}
\end{array}\right)
$$

For $2 \leq m \leq M, \mathbf{h}_{1, m}, \mathbf{h}_{2, m}$ are $N_{s}$-column vectors of which elements are expressed as

$$
\begin{aligned}
& \mathbf{h}_{1, m}[i]=\sum_{k=2}^{L} u\left[\Delta_{k}-i\right] \gamma_{1, k}\left[n_{m}+i\right] c_{d}\left[N_{s}-\Delta_{k}+i\right] h_{k}(7) \\
& \mathbf{h}_{2, m}[i]=\sum_{k=1}^{L} u\left[i-\Delta_{k}-1\right] \gamma_{1, k}\left[n_{m}+i\right] c_{d}\left[i-\Delta_{k}\right] h_{k}
\end{aligned}
$$

where $i=1, \ldots, N_{s}, \gamma_{1,1} \equiv 1, \quad \Delta_{1}=0$, and $u[i]$ is an unit step function. $\mathbf{h}_{1, m}$ includes the effect of the previous symbol to the current observation and $\mathbf{h}_{2, m}$ represents the effect of the current symbol to the observation.

\section{PROJECTION-BASED CHANNEL ESTIMATION}

For long code CDMA systems, several channel estimation schemes are proposed[1] [6]. In this section, based on projection method, we present a semi-blind channel estimation technique for long code CDMA systems using data from a single slot. This method eliminates the data part $\mathcal{H}(\mathbf{h}) \mathbf{s}_{d}$ in eq.(1) by projecting the observation vector to the orthogonal complement of the filtering matrix $\mathcal{H}(\mathbf{h})$. Since the unknown channel parameter $\mathbf{h}$ is incorporated in the filtering matrix $\mathcal{H}(\mathbf{h})$, it is impossible to get the orthogonal complement of the matrix $\mathcal{H}(\mathbf{h})$ directly without knowing the channel parameter $\mathbf{h}$. The filtering matrix $\mathcal{H}(\mathbf{h})$, fortunately, is block lower bidiagonal since the delay spread is assumed to be less than one symbol duration. Furthermore, since the previous symbol does not affect observation after the largest propagation path delay, the elements of vector $\mathbf{h}_{1, m}$ in eq.(5) are zero after $\Delta_{L}$ chip-rate samples. The element of the vector $\mathbf{h}_{2, m}$ is a linear combination of the signals of all propagation paths after $\Delta_{L}$. Decimating the first $\Delta_{L}$ chip-rate samples from observations during each symbol interval, we obtain the modified filtering matrix $\tilde{\mathcal{H}}(\mathbf{h})$ of dimension $M\left(N_{s}-\Delta_{L}\right) \times M$, which is expressed as

$$
\tilde{\mathcal{H}}(\mathbf{h})=\operatorname{diag}\left(\tilde{\mathbf{h}}_{2,1}, \ldots, \tilde{\mathbf{h}}_{2, M}\right)
$$

where $\tilde{\mathbf{h}}_{2, m}$ is a $\left(N_{s}-\Delta_{L}\right)$-column vector which is represented as 


$$
\tilde{\mathbf{h}}_{2, m}=\Gamma_{m} \mathbf{h}
$$

where the entries of $\Gamma_{m}$ are given as

$$
\Gamma_{m}[i, j]=\gamma_{1, j}\left[(m-1) N_{s}+\Delta_{L}+i\right] c_{d}\left[\Delta_{L}-\Delta_{j}+i\right]
$$

$, i=1, \ldots, N_{s}-\Delta_{L}, j=1, \ldots, L$

Proposition 1 (Decomposition) The first $\Delta_{L}$ row decimated filtering matrix $\tilde{\mathcal{H}}(\mathbf{h})$ has the following decomposition as a form of composite linear transformation.

$$
\tilde{\mathcal{H}}(\mathbf{h})=\mathbf{H}\left(I_{M} \otimes \mathbf{h}\right)
$$

where $\otimes$ is the Kronecker product, $I_{M}$ is a $M \times M$ identity matrix, $\mathbf{h}=\left[h_{1}, \ldots, h_{L}\right]^{T}$, and the matrix $\mathbf{H}$ is determined by only known data and expressed as

$$
\mathbf{H}=\operatorname{diag}\left(\mathbf{H}_{2,1}, \ldots, \mathbf{H}_{2, M}\right)
$$

and the submatrix $\mathbf{H}_{2, m}$ is $\Gamma_{m}$ defined in eq.(11).

$\mathbf{H}$ is a tall matrix since the number of decimated rows is much smaller than the spreading gain and is full column rank almost surely for the practical range of spreading gain since the columns of submatrix $\mathbf{H}_{2, m}$ are nonzero and independent due to the fact that they are determined by the channelization codes and the delayed random scrambling sequence $c_{s, 1}$ as shown in eq.(11). The loss of signal power due to decimation of the first $\Delta_{L}$ chip-rate observation samples for the decomposition is not significant since the delay spread is much less than one symbol duration.

The modified data model is expressed as

$$
\tilde{\mathbf{y}}=\tilde{\mathcal{T}}\left(\mathbf{s}_{p}\right) \mathbf{h}+\mathbf{H}\left(I_{M} \otimes \mathbf{h}\right) \mathbf{s}_{d}+\tilde{\mathbf{n}}
$$

where $\tilde{\mathbf{y}}$ is the row decimated version of $\mathbf{y}, \tilde{\mathcal{T}}\left(\mathbf{s}_{p}\right)$ is the row decimated of $\mathcal{T}\left(\mathbf{s}_{p}\right)$, and $\tilde{\mathbf{n}}$ is an i.i.d. circularly symmetric Gaussian noise vector.

The columns of matrix $\mathbf{H}$ span the signal subspace of dimension $L$ and the basis of the orthogonal complement of $\mathbf{H}$ is obtained using singular value decomposition and and forms the columns of the matrix $\mathbf{G}$. Any column vector in $\mathbf{G}$ is orthogonal to the column vectors of $\mathbf{H}$. We have

$$
\mathbf{G}^{H} \mathbf{H}=0
$$

projecting the modified observation vector in eq.(14) on the orthogonal complement of $\mathbf{H}$, we obtain

$$
\mathbf{G}^{H} \tilde{\mathbf{y}}=\mathbf{G}^{H} \tilde{\mathcal{T}}\left(\mathbf{s}_{p}\right) \mathbf{h}+\mathbf{G}^{H} \tilde{\mathbf{n}}
$$

where $\mathbf{G}^{H} \tilde{\mathbf{n}}$ is Gaussian noise with zero mean and covariance matrix $\Sigma=\mathbf{G}^{H} \mathbf{G}$. The data model in eq.(16) is a general linear model and is solved by a standard least square algorithm.

\section{NUMERICAL RESULTS}

The performance of the proposed method is analyzed through the Cramér-Rao bound(CRB) and the Monte Carlo simulation and compared with the training-only estimation. The effects of the various parameters in code division multiple access systems such as spreading gain, number of pilot symbols, power ratio of pilot to data channel, and the signal to noise ratio(SNR) are considered. The SNR is defined as $\frac{\|\mathbf{h}\|^{2}\left(1+G^{2}\right) N_{s}}{\sigma_{n}^{2}}$. The channel used for simulation is a two tap channel with 3 chip delay and its coefficient is $\mathbf{h}=\left[1,0,0, \exp \left(-j \frac{\pi}{4}\right)\right]^{T}$. The noise variance $\sigma_{n}^{2}$ is assumed to be known. The scrambling code used for simulation is a realization of random code. For the channelization codes, two Walsh-Hadamard codes with the same length are used. The pilot sequence is prefixed and the data sequence is randomly generated for each slot.

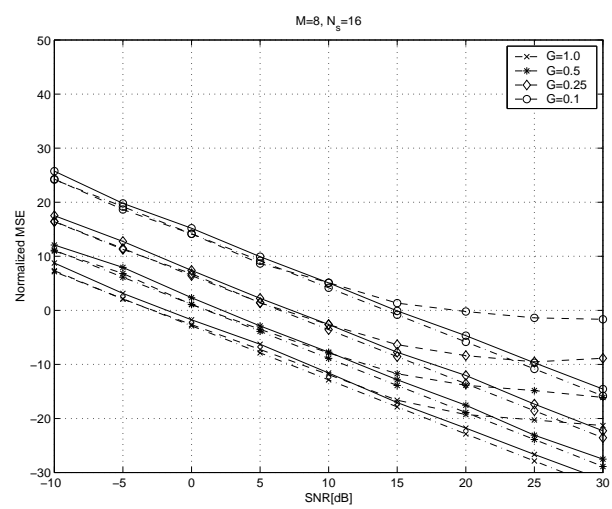

Fig. 2. Normalized mean square error versus SNR for $\mathrm{M}=8$ (solid line:projection-based ,dotted line:training-only, dashed-dotted lin: CRB)

Figure 2 shows the normalized mean square error of the training-only estimation, the proposed method, and CRB for the case of 8 pilot symbols and spreading factor of 16 . We observe that the performance of the training-only method degrades significantly from CRB as the SNR increases regardless of the pilot gain. At the low SNR, the additive noise is dominant over the unknown data channel. However, the effect of the unknown data channel on the trainingonly estimator becomes dominant at the high SNR. Thus, the training-only estimation shows a performance limit as SNR increases. The proposed method shows no performance degradation at medium and high SNR since it eliminates the unknown data interference effectively. Its performance is slightly worse than CRB due to the signal power loss by the decimation of the first $\Delta_{L}$ chip-rate samples. However, the difference is negligible when spreading gain is higher.

Figure 3 shows the normalized mean square error as a function of spreading gain at $20 \mathrm{~dB}$ SNR and pilot gain of 
0.25. Actual operation range of SNR is considered to be higher than $10 \mathrm{~dB}$ in this definition of SNR to have bit error rate lower than $10^{-3}$. As shown in figure 3 , both of the proposed method and the training-only estimation approach the CRB as the spreading gain increases[5]. However, the performance of training-only estimation is much worse than $\mathrm{CRB}$ at low spreading gain where the performance improvement by the projection-based method is significant. The noticeable deviation of the proposed method from CRB at low spreading gain is due to the relative high signal power loss due to the decimation.

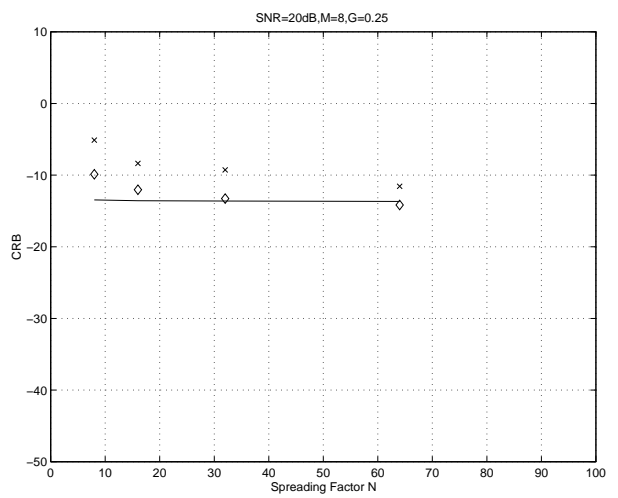

Fig. 3. Normalized mean square error versus spreading factor at $\mathrm{SNR}=20 \mathrm{~dB}$ (solid line:projection-based ,dotted line:training-only, dashed-dotted lin: $\mathrm{CRB}$ )

\section{DISCUSSION}

In this paper, the proposed method is applied to the single user scenario by assuming that the $\mathbf{n}$ includes other user interference. However, it can be extended to multiuser cases. Consider, for example, when there are $K$ users. We then have the following data model

$$
\mathbf{y}=\sum_{k=1}^{K}\left[\mathcal{T}_{k}\left(\mathbf{s}_{p_{k}}\right) \mathbf{h}_{k}+\mathcal{H}_{k}\left(\mathbf{h}_{k}\right) \mathbf{s}_{d_{k}}\right]+\mathbf{n}
$$

Assuming the timing is acquired for all users at the receiver, we obtain a similar decomposition by decimating the observation samples overlapped by two adjacent symbols of each user. For asynchronous transmission, the number of required decimation blocks is the same as the number of users in general. After proper decimation of observation samples, we have

$$
\begin{aligned}
\tilde{\mathbf{y}} & =\left[\tilde{\mathcal{T}}_{1}\left(\mathbf{s}_{p_{1}}\right) \ldots \tilde{\mathcal{T}}_{K}\left(\mathbf{s}_{p_{K}}\right)\right]\left[\begin{array}{c}
\mathbf{h}_{1} \\
\vdots \\
\mathbf{h}_{K}
\end{array}\right] \\
& +\left[\mathbf{H}_{1} \ldots \mathbf{H}_{K}\right]\left[\begin{array}{c}
\left(I_{M} \otimes \mathbf{h}_{1}\right) \mathbf{s}_{d_{1}} \\
\vdots \\
\left(I_{M} \otimes \mathbf{h}_{K}\right) \mathbf{s}_{d_{K}}
\end{array}\right]+\tilde{\mathbf{n}}
\end{aligned}
$$

Thus, we can obtain the orthogonal space of the concatenated filtering matrix $\left[\begin{array}{llll}\mathbf{H}_{1} & \ldots & \mathbf{H}_{K}\end{array}\right]$. As the number of users increases, the concatenated filtering matrix becomes less overdetermined which makes applying the proposed method difficult in many user case. However, when the number of users is small and the power control between users is not available, the proposed method is very useful since it effectively eliminates other user interference.

\section{CONCLUSION}

A new semi-blind channel estimation technique based on projection method is proposed for long code CDMA systems which employ code division multiplexed pilot symbols. The conventional training-only channel estimation applied to CDMA systems is shown to have performance limit at medium and high SNR with a finite spreading gain. The proposed projection-based channel estimation overcomes this effect. This method can be extended to multiuser scenario especially when there are a small number of users and the power control is not available. It can also be used as an initialization technique for high performance iterative techniques such as the maximum likelihood approach.

\section{REFERENCES}

[1] Stefano Buzzi and H.Vincent Poor. "Channel Estimation for Multirate/Multicode DS/CDMA Systems with Long Spreading Codes". In Wireless Communications and Networking Conference,2000.WCNC. 2000 IEEE, volume 3, pages 1142-1146, 2000.

[2] Elisabeth De Carvalho and Dirk T.M. Slock. "CramerRao bounds for semi-blind, blind and training sequence based channel estimation". In Proc. IEEE SP Workshop on SPAWC, pages 129-132, Paris, April 1997.

[3] Harri Holma and Antti Toskala. WCDMA for UMTS. Wiley, New York, NY, 2001.

[4] Fuyun Ling. "Optimal Reception,Performance Bound, and Cutoff Rate Analysis of Reference-Assisted Coherent CDMA Communications with Applications". IEEE Trans.Communications, COM-47(10):15831592 , Oct 1999.

[5] Xavier Mestre and Javier R. Fonollosa. "Asymptotic Performance of ML Channel Estimators in WCDMA Systems: Randomized Codes Approach". In IEEE Intl. Conf. Acoust. Speech, Sig. Proc., Salt Lake City, UT, May 2001.

[6] Zhengyuan Xu and Michail K.Tsatanis. "Blind Channel Estimation for Long Code Multiuser CDMA Systems". IEEE Trans.Signal Processing, SP-48(4):9881001, April 2000. 